\title{
Type 3 Diabetes Reflects Disordered Lipid Metabolism in the Human Brain Related to Higher Degree of Unsaturated Fatty Acids Composition and is not Related to Body Mass Index
}

\author{
Vincent van Ginneken ${ }^{1 *}$, Evert de Vries ${ }^{1}$, Verheij $E^{2}$ and Jan van der Greef ${ }^{2,3}$ \\ ${ }^{1}$ Blue Green Technologies, Runderweg 6, 8219 PK Lelystad, The Netherlands \\ ${ }^{2}$ Netherlands Organisation for Applied Scientific Research (TNO), Utrechtseweg, AJ Zeist, The Netherlands \\ ${ }^{3}$ Sino-Dutch Center for Preventive and Personalized Medicine, Leiden University, RA Leiden, The Netherlands
}

\begin{abstract}
In this study we investigated lipid profiles of brain of post mortem type 2 diabetes patients T2DM elderly patients in comparison to a Control group (Co) of the "Netherlands Brain Bank" using LCMS techniques. Here we report that brains of these T2DM patients contain more double bonds and consequently are more rigid. In a small cohort $(\approx 200$ patients) we prove that these brain diseases are not interrelated with BMI so obesity is not a major cause. The predicted wave of brain diseases of mild-Alzheimer (m-AD), dementia and depression of the "baby boom generation" might be evolved due to the "fatty" and more "rigid" brain structure due to the quality of fats eaten during earlier lifespan. Our major conclusion is that diabetes and its treatment among T2DM patients are more associated with structural disturbances (lipid composition) in the brain than with glycaemic control. Therefore we introduce the new terminology "Type 3 diabetes" (T3DM) referring to the mental disorders as a consequence of a disordered lipid metabolism in the human brain related to higher degree of unsaturated fatty acids composition.
\end{abstract}

Keywords: Human brain; Post-mortem; Type 2 diabetes; Mental disorders; Mild-Alzheimer; Dementia

\section{Introduction}

The incidence of diabetes mellitus (T2DM) is increasing at an alarming rate and has become a major public health concern worldwide [1]. In addition, emerging evidence suggests that diabetes affects cognitive function and increases the incidence of dementia [2-5] and "mild-Alzheimer Disease (m-AD)" [4,6,7]. Recent studies indicate that glycaemic control is implicated in the development of cognitive dysfunction/impairment [2]. In this study we investigated lipid profiles of brain of post mortem type T2DM elderly patients in comparison to a Control group (Co) using LCMS techniques as earlier performed $[8,9]$. Here we report that brains of these T2DM patients contain more double bonds and consequently are more rigid [10]. In this respect, notify that a saturated fatty acid is fully loaded with hydrogen atoms and contains only single bonds between its carbon atoms while in an unsaturated fat two hydrogens were missing from the middle of the carbon chain and therefore the two carbons form a double bond which makes the molecule more rigid [10].

In a small cohort $(\approx 200$ patients $)$ of the Netherlands Brain association we prove that these brain diseases are not interrelated with BMI so obesity is not a major cause. The predicted wave of brain diseases of mild-Alzheimer (m-AD), dementia and depression of the "baby boom generation" [7] might be evolved due to the "fatty" and more "rigid" brain structure due to the quality of fats eaten during earlier lifespan. Our major conclusion is that diabetes and its treatment among T2DM patients are more associated with structural disturbances (lipid composition) in the brain than with glycaemic control.

These new insights of diabetes-3 [11] might abate this brain lipid disorder related to the saturation degree of the human elderly brain and could provide plausible opportunity for further investigation by pharmaceutical industry of relationship of related disease for the design of drugs like is already performed for mild-Alzheimer [12] and Dementia [13] and development of new therapies in order to prevent mild-Alzheimer disease [14]. Such treatment of patients could be performed with drugs purporting to act on molecular targets of the lipid metabolism in combination with quality of functional foods in relation to quality and saturation degree of fats eaten during adolescence $[10,15]$.

Patients with Type 2 Diabetes Mellitus (T2DM) usually also are at increased risk for cognitive impairment and dementia [2,5]. In addition, elderly people with T2DM are more prone to develop cognitive impairment, for which several potential risk factors have been proposed. In the comparison of healthy elderly (Control) and T2DM a range of vascular, metabolic, and psychosocial risk factors, are involved leading to cerebrovascular damage (or both), poor glycemic control, hypoglycemia, microvascular disease, inflammation, and depression [15-17]. Recent studies indicate that glycaemic controlmainly Postprandial Glucose (PPG) is implicated in the development of cognitive dysfunction [18]. Insulin dysregulation and hyperglycaemia may play an important role in neurodegeneration $[2,18]$. Poor glycemic control is in most studies stipulated as the viscous circle of bidirectional associations between dementia and hypoglycaemia [2,5,16]. However, the effects of recurrent moderate hypoglycemia on cognitive decline and dementia remain largely uninvestigated [5].

Cognitive impairments in patients with T2DM have been associated with vascular risk factors, such as hypertension and dyslipidemia, and with diabetes-related factors, such as glycemic control [16] duration of the disease and treatment modality $[12,13,18,19]$. It may be due

${ }^{*}$ Corresponding author: Vincent van Ginneken, Blue Green Technologies, Runderweg 6, 8219 PK Lelystad, The Netherlands, Tel: 310638071180; E-mail: vvanginneken@hotmail.com

Received June 03, 2017; Accepted June 20, 2017; Published June 27, 2017

Citation: Ginneken VV, Vries Ede, Verheij E, Greef JVD (2017) Type 3 Diabetes Reflects Disordered Lipid Metabolism in the Human Brain Related to Higher Degree of Unsaturated Fatty Acids Composition and is not Related to Body Mass Index. J Bioanal Biomed 9: 159-163. doi:10.4172/1948-593X.1000171

Copyright: ( 2017 Ginneken VV, et al. This is an open-access article distributed under the terms of the Creative Commons Attribution License, which permits unrestricted use, distribution, and reproduction in any medium, provided the original author and source are credited. 
Citation: Ginneken VV, Vries Ede, Verheij E, Greef JVD (2017) Type 3 Diabetes Reflects Disordered Lipid Metabolism in the Human Brain Related to Higher Degree of Unsaturated Fatty Acids Composition and is not Related to Body Mass Index. J Bioanal Biomed 9: $159-163$. doi:10.4172/1948-593X.1000171

to degenerative change, circulatory disease, senile dementia which results in a progressive loss of mental faculties such as memory and orientation, by lowered performance on several cognitive domains, depression and $\mathrm{A} \beta /$ tau-independent mechanism such as mildAlzheimer disease (m-AD) [4,6,7] and depression [15,17]. Some of the "brain-diseases" as a consequence of T2DM are associated with the occurrence of well-described microvascular complications and can be associated with slowly progressive brain damage often referred as "diabetic encephalopathy" and are characterized by mild to moderate impairments in cognitive functioning [18]. The magnitude of these cognitive deficits appears mild to moderate, but can significantly hamper daily functioning in processes as attention and executive functioning, information processing, speed and memory, in this way adversely affecting quality of life [20]. So probably "Diabetic encephalopathy", is a term that encompasses functional impairment of cognition, cerebral cognition, cerebral signal conduction, neurotransmission and synaptic plasticity, and underlying structural pathology associated with T2DM. These mental disorders such as dementia, $\mathrm{m}-\mathrm{AD}$ and depression as a consequence of Type 2 diabetes were also recently termed Type 3 diabetes [11].

Despite the fact that a whole reductionistic array of transgenic mouse models has been developed in order to study m-AD $[6,21]$ the primarily cause remains unknown. It is not known whether these brain diseases develop as a result of aberrant lipid metabolism [22]. In order to develop a mechanistic insight and to identify the factors that initiate and promote early disturbances in the nervous system [23], here we perform a new approach based on a Systems Biology lipidomics approach of post mortem material of the "Dutch Brain Bank". This approach isot new to us because earlier we performed similar LCMS techniques in a C57bl6 High-Fat diet (HF) induced mouse model in order to find biomarkers for early diabetes (Insulin Resistance) or T2DM $[8,9]$. Such LCMS brain studies at post mortem human brain material are crucial to understanding what specific factors or thresholds in diet and metabolism must be achieved to promote biologically significant changes in the nervous system. In addition due to the scarceness of human brain banks in combination with advanced LCMS techniques such lipidomics based LCMS techniques at human brain are rare. In literature solely membrane phospholipid abnormalities have been observed in brain tissue of schizophrenic patients when compared to a control group [24]. Studies on brain tissue of T2DM patients have so far not been reported. To date, its pathogenic mechanism is largely unclear. Lipids play a key role in determining membrane fluidity, and changes in lipid and fatty acids composition have been reported to alter important cellular functions $[24,25]$. A decreased membrane polysaturation may act to decrease the activity of the major energy-consuming processes of the cell such as reducing the flux of ions and protons and subsequently the energy needed to maintain ionic homeostasis. Membrane fatty acid composition influences insulin action via alteration of membrane proteins specifically associated with the action of insulin. Such mechanism would include modulation both of receptor affinities and translocation to the membrane of nutrient transporters [25]. We recently observed based on ration of 16:0 to 18:0 fatty acids of brain homogenate that the saturation index for the Cholesterylesters for 16:0 was significantly higher in white matter of T2DM patients in comparison to white matter of the control group $(\mathrm{P}<0.05)[8]$. Hence, in this manuscript we hypothetisize that there are structural changes in lipid brain composition of a T2DM patient group which can explain all earlier mentioned characteristics of diabetic encephalopathy [20]. The aim of this study is to compare the biochemical lipid composition in brain white and red matter between T2DM patients with controls.

\section{Material and Methods}

\section{Experimental set-up}

This study encompasses precision together with robustness because at first all selected eight T2DM patients were classified based on their patient documentation in combination with a fructosamine test which was significantly $(\mathrm{P} \leq 0.04)$ increased in the T2DM group [8]. Fructosamine gives an estimation of average glycaemic control over the previous 2-3 weeks [26].

Brain material of Patients was post-mortem histopathologically controlled for Alzheimer for amyloid stage according to Braak criterium $[8,27]$. For Alzheimer stage 1 there is no accumulation of abnormally phosphorylated tau protein. For stage 5 abnormally accumulation of this protein can be observed, a hallmark of Alzheimer disease [27]. All patients were in stages varying between $0-2$ with exception of patient Co-7 which was at stage 3 [8].

Reversed phase liquid chromatography coupled to mass spectrometry (LC-MS) was used to quantify and qualify the rearrangement and repartitioning of fat stores in the brain of male post mortem subjects $(\mathrm{CO} n=8 ; 73.5 \pm 10.5$ years; T2DM, $n=8 ; 77.3 \pm 7.2$ years) which were obtained from the 'Netherlands Brain Bank'[8].

\section{LCMS measurements}

We quantified 109 lipid compounds, including Cholesterylesters (ChE), Lysophosphatidyl-cholines (LPC), Phosphatidyl-choline (PC), Sphingomyelin (SPM), triacylglycerols (TG), diacylglycerols (DG) and Plasmalogens (PG) [8]. Lipids and Free Fatty Acids (FFA) were analyzed with electrospray LC-MS as earlier performed [8]. For blood plasma 10 $\mu \mathrm{l}$ samples were extracted with $300 \mu \mathrm{l}$ of Isopropanol (IPA) containing several internal standards (IS: C17:0 lysophosphatidylcholine, di-C12:0 phosphatidylcholine, tri-C17:0 glycerol ester, C17:0 cholesterol ester and heptadecanoic acid (C17:0)). Fifty $\mu \mathrm{L}$ of the brain homogenate was mixed with $1000 \mu \mathrm{L}$ IPA containing 4 internal standards. After mixing and centrifugation the supernatant was transferred to an autosampler vial. Lipids were separated on a $150 \times 3.2 \mathrm{~mm}$ i.d. C4 Prosphere column (Alltech, USA) using a methanol gradient in $5 \mathrm{mM}$ ammonium acetate and $0.1 \%$ formic acid (mobile phase A: $5 \%$ methanol, mobile phase B: $90 \%$ methanol). The flow rate was $0.4 \mathrm{ml} / \mathrm{min}$ and the gradient was as follows: $0-2 \mathrm{~min}-20 \% \mathrm{~B}, 2-3 \mathrm{~min}-20 \%$ to $80 \% \mathrm{~B}, 3-15 \mathrm{~min}-80 \%$ to $100 \%$ B, 15-25 min-hold $100 \%$ B, 25-32 min-condition at 20\% B.

The instrument used was a Thermo LTQ equipped with a Thermo Surveyor HPLC pump. Data were acquired by scanning the instrument from m/z 300 to 1200 at a scan rate of approximately 2 scans/s in positive ion ESI mode.

The FFA LC-MS platform employs the same sample and similar HPLC conditions as the lipid method. The ammonium acetate concentration is $2 \mathrm{mM}$ instead of $5 \mathrm{mM}$ and no formic acid was added. The gradient: $0-2 \mathrm{~min}-30 \% \mathrm{~B}, 2-3 \mathrm{~min}-30 \%$ to $70 \% \mathrm{~B}, 3-10 \mathrm{~min}-70 \%$ to $100 \% \mathrm{~B}, 10-15$ min-hold $100 \% \mathrm{~B}, 15-20$ min-condition at $30 \% \mathrm{~B}$. Detection of FFA is performed in negative ion ESI mode. Combined the two methods provide (semi)quantitative data for approximately 200 different identified lipids and FFA.

Each extract was injected three times $(10 \mu \mathrm{l})$, once for the LC-MS FFA platform and two times for the LC-MS lipid platform. Furthermore, a Quality Control (QC) sample was prepared by pooling the samples. The pool was divided into $10 \mu$ aliquots that were extracted the same as the study samples. The QC samples were placed at regular intervals in the analysis sequence (one QC after every 10 samples). The QC samples 
Citation: Ginneken VV, Vries Ede, Verheij E, Greef JVD (2017) Type 3 Diabetes Reflects Disordered Lipid Metabolism in the Human Brain Related to Higher Degree of Unsaturated Fatty Acids Composition and is not Related to Body Mass Index. J Bioanal Biomed 9: $159-163$. doi:10.4172/1948-593X.1000171

served two purposes. The first is a regular quality control sample to monitor the LC-MS response in time. After the response has been characterized, the QC samples were used as standards of unknown composition to calibrate the data.

In plasma samples, the 6 dominant lipid classes observed with these two methods are the lyso-phosphatidylcholines (IS used: C17:0 lyso-phosphatidylcholine), phosphatidylcholines (IS used: di-C12:0 phosphatidylcholine), sphingomyelines (IS used: di-C12:0 phosphatidylcholine), cholesterylesters (IS used: C17:0 cholesterylester), triacylglycerols (IS used: tri-C17:0 glycerol ester), and free fatty acids (IS used: C17:0 FFA) (Figure 1A and 1B). In addition to these lipids, the extracts also contain minor lipids, but these were either not detected (concentration too low relative to very abundant lipids like phosphatidylcholines and diacylglycerols, DG) or they were not included in data processing with exception of the DG. The LC-MS lipid and LC-MS FFA data were processed using the LC-Quan software (Thermo).

Analysis of plasmalogens: Analysis of Cholesterylesters (ChE), Lysophosphatidyl-cholines (LPC), Phosphatidylcholine (PC),

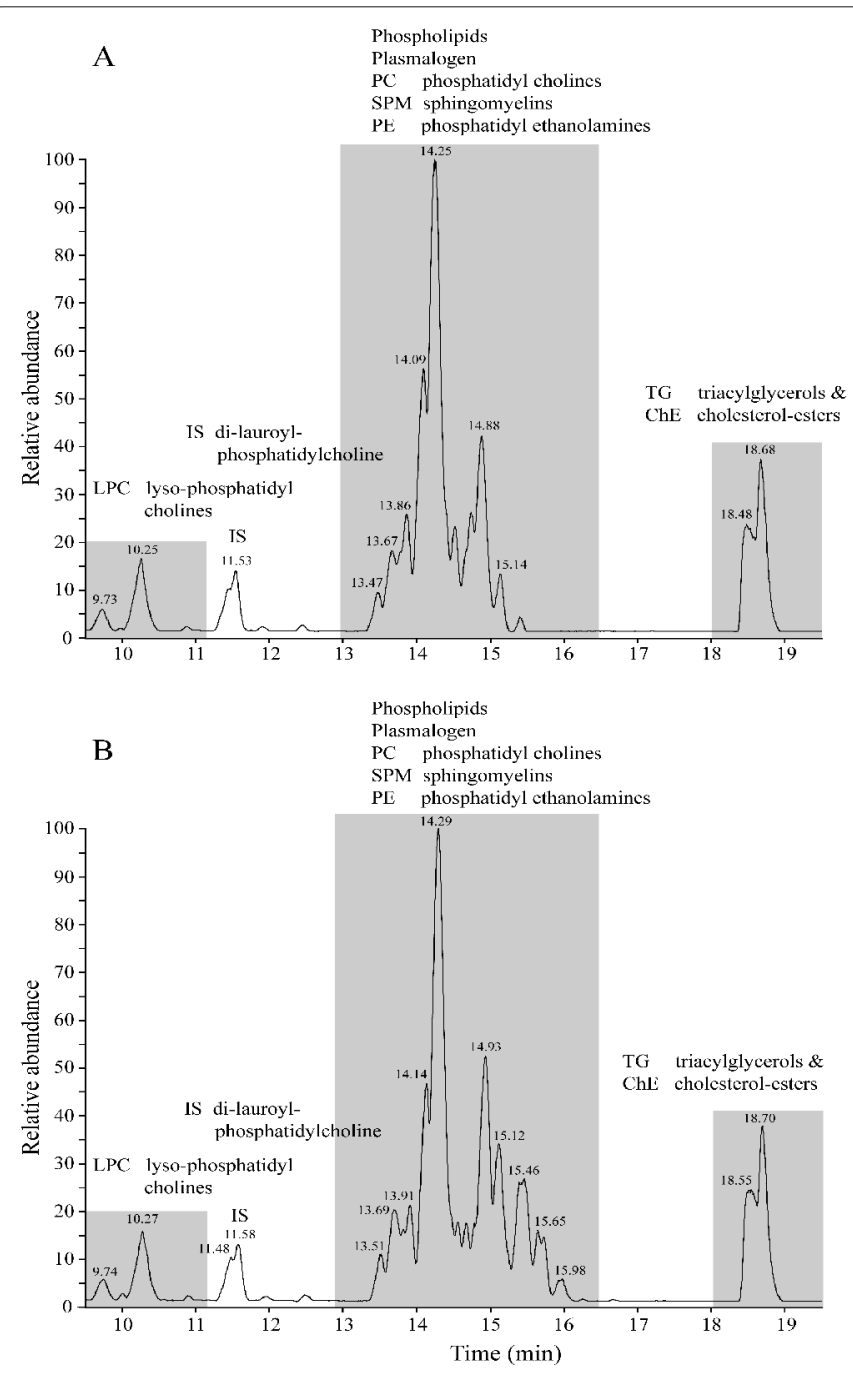

Figure 1: Using reversed phase liquid chromatography coupled to massspectrometry (LC-MS) a chromatogram was obtained of the brain-homogenate of a Co-patient no. 1 (Figure 1A) and a type-2 (T2DM) patient (Source: 8).
Sphingomyelin (SPM) and Triacylglycerols (TG) were based on molecular mass and retention time using internal standards. Because no standards for the plasmalogens exist, mass lists determined by using Atmospheric Pressure Chemical Ionization (APCI) and Electrospray Ionization (ESI) mass spectrometry (MS) techniques were used. These mass lists were published on internet www.byrdwell.com/plasmogens and www.byrdwell.com/-PhosphatidylEthanolamine.

\section{Statistics}

For all measured parameters the mean value of the control postmortem human group was compared to the mean value of the postmortem T2DM human group. Statistics were performed via SPSS using one-way ANOVA for differences between control and T2DM groups. $\mathrm{P}$ $\leq 0.05$ was considered as statistically significant. Normality of the data and homogeneity of variances were checked by Kolmogorov-Smirnov and $\mathrm{F}_{\max }$ tests, respectively $[8,9]$.

\section{Results}

Using reversed phase Liquid Chromatography Coupled to Massspectrometry (LC-MS) a chromatogram was obtained of the brainhomogenate of a Co-patient no. 1 (Figure 1A). Clearly visible are three groups of chemical compounds:

A. 9-11 min retention time Lyso-phosphatidylcholines (LPC) with at $11.5 \mathrm{~min}$ the Internal Standard di-lauroyl-phoshatidylcholine.

B. 13-16 min Plasmalogens, Phosphatidylcholines (PC), Sphingomyelins (SPM) and Phosphatidylethanolamines (PE).

C. 17-19 min Triacylglycerols (TG) and Cholesteryl-esters (ChE). The chromatogram of a T2DM patient no.1 shows a similar pattern (Figure 1B).

Here we report in Table 1 that brains of these T2DM patients were more saturated and consequently more rigid due to significantly more double bonds in the red matter for LPC C:18 $\left(\mathrm{P}<0.0349^{*}\right)$ and TG C:50 $\left(\mathrm{P}<0.0239^{*}\right)$ while for white matter this was for TG C:50 $\left(\mathrm{P}<0.0239^{*}\right)$.

\section{Discussion and Conclusion}

Our major conclusion of this manuscript is that we have found a plausible mechanistic explanation related to the lipid composition of elderly with type-2 diabetes in relation to the earlier described cognitive impairment (depression, dementia, $\mathrm{m}-\mathrm{AD}$ ) which probably in the past is indicated with the one decade ago correctly chosen term "diabetic encephalopathy" $[8,20]$, but which we now replace by the term "type3 diabetes" [11]. Increasing the content of polyunsaturated fatty acids within cell membranes would increase the membrane fluidity, the number of insulin receptors and the action of insulin for muscle tissue. A converse effect occurs when the concentration of saturated fatty acids in the membranes is increased [25] like we earlier described (Table 1). In this respect a difference should be made related to "traditional" Alzheimer determined by genetic predisposition which manifest at young/intermediate age [8,27]. In order to gain a better understanding of $\mathrm{m}$-AD pathogenesis among the elderly we urgently need at first acknowledge this dichotomous definition of Alzheimer diseases in general.

From Figure 2 we can conclude histopathological post-mortem determined brain diseases of the elderly are not related to Body Mass Index (BMI) because the BMI-curve is not skewed to the right e.g. a $\mathrm{BMI}$ of 30 which is defined as obese. So we can make the statement that "brain diseases of the elderly" including dementia and $\mathrm{m}-\mathrm{AD}$ are more 
Citation: Ginneken VV, Vries Ede, Verheij E, Greef JVD (2017) Type 3 Diabetes Reflects Disordered Lipid Metabolism in the Human Brain Related to Higher Degree of Unsaturated Fatty Acids Composition and is not Related to Body Mass Index. J Bioanal Biomed 9: $159-163$. doi:10.4172/1948-593X.1000171

\begin{tabular}{|c|c|c|c|c|c|c|c|}
\hline \multirow{2}{*}{$\begin{array}{c}\text { Lipid } \\
\text { Comp. }\end{array}$} & \multirow{2}{*}{$\begin{array}{l}\text { Number } \\
\text { C-atoms }\end{array}$} & \multicolumn{2}{|c|}{ Red matter } & \multicolumn{2}{|c|}{ White matter } & \multirow[b]{2}{*}{ A vs. B } & \multirow[b]{2}{*}{ C vs. D } \\
\hline & & Control (A) & Diabetes (B) & Control (C) & Diabetes (D) & & \\
\hline LPC & $C: 18$ & $206.3 \pm 23.43$ & $235.1 \pm 25.80$ & $92.6 \pm 32.68$ & $120.8 \pm 46.20$ & $0.035^{*}$ & 0.183 \\
\hline \multirow[t]{3}{*}{ SPM } & $C: 23$ & $6.6 \pm 5.65$ & $8.00 \pm 10.82$ & $166.0 \pm 67.11$ & $185.4 \pm 101.78$ & 0.752 & 0.660 \\
\hline & $C: 24$ & $15.7 \pm 8.11$ & $14.9 \pm 3.47$ & $30.7 \pm 9.92$ & $31.6 \pm 13.15$ & 0.826 & 0.882 \\
\hline & $C: 25$ & $13.8 \pm 3.12$ & $16.3 \pm 2.73$ & $41.3 \pm 15.25$ & $44.8 \pm 19.29$ & 0.105 & 0.691 \\
\hline DG & $C: 34$ & $35.3 \pm 6.48$ & $36.0 \pm 13.11$ & $12.2 \pm 3.88$ & $13.6 \pm 4.52$ & 0.893 & 0.511 \\
\hline \multirow[t]{2}{*}{ PC } & $C: 32$ & $277.7 \pm 31.56$ & $259.4 \pm 25.29$ & $109.9 \pm 34.27$ & $115.1 \pm 53.53$ & 0.224 & 0.819 \\
\hline & $C: 34$ & $18.9 \pm 1.09$ & $18.61 \pm 0.78$ & $6.7 \pm 2.77$ & $6.9 \pm 4.70$ & 0.507 & 0.889 \\
\hline$P E$ & $C: 34$ & $46.3 \pm 3.00$ & $48.0 \pm 5.45$ & $45.2 \pm 4.32$ & $47.1 \pm 4.86$ & 0.452 & 0.422 \\
\hline \multirow[t]{2}{*}{ ChE } & $C: 16$ & $58.2 \pm 7.31$ & $67.0 \pm 10.07$ & $54.2 \pm 7.07$ & $70.1 \pm 9.07$ & 0.068 & 0.002 \\
\hline & $C: 18$ & $38.3 \pm 6.05$ & $33.9 \pm 5.64$ & $34.3 \pm 10.26$ & $35.8 \pm 5.48$ & 0.151 & 0.727 \\
\hline \multirow[t]{2}{*}{ TG } & $C: 48$ & $61.5 \pm 12.80$ & $57.2 \pm 11.47$ & $54.7 \pm 19.26$ & $49.7 \pm 13.08$ & 0.493 & 0.555 \\
\hline & $C: 50$ & $26.7 \pm 4.37$ & $20.5 \pm 5.31$ & $23.4 \pm 5.72$ & $16.6 \pm 5.74$ & $0.024^{*}$ & $0.033^{*}$ \\
\hline
\end{tabular}

Table 1: Saturation degree according to Hulbert et al. [25] of the several lipid compounds of postmortem neo-cortical tissue divided in red and white matter.
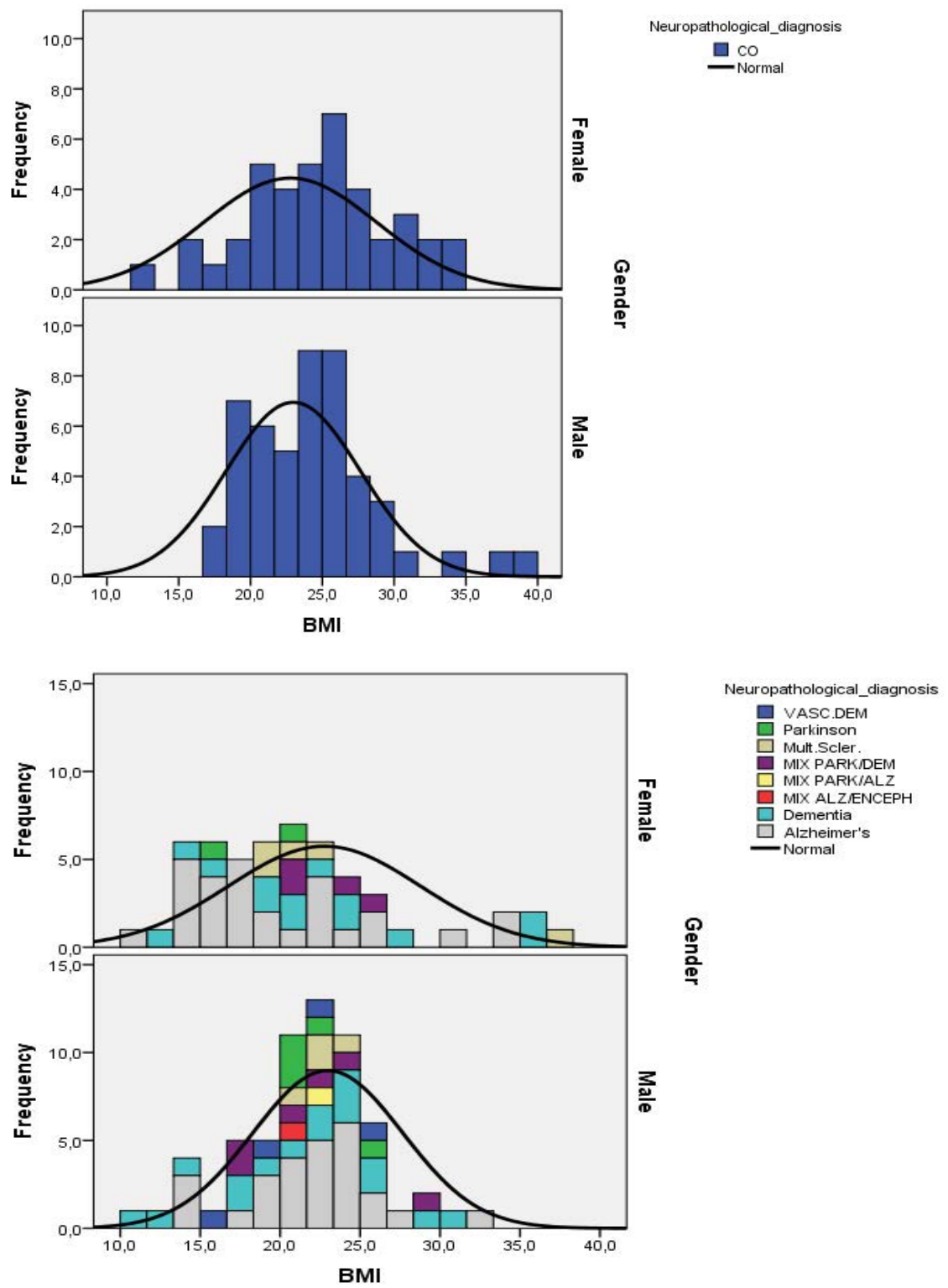

Figure 2: Frequency distribution of postmortem patient's cohort $(n=203)$ of the "Netherlands Brain Association" showing the number of patients with Neuropathological disorders by Body Mass Index (BMI). Figure 2A [Top]: Control group exist out of $n=49$ males and $n=41$ females; Figure 2B: [Bottom] while the group with postmortem histopathological determined "brain diseases" consist out of $n=62$ males and $n=51$ females. 
Citation: Ginneken VV, Vries Ede, Verheij E, Greef JVD (2017) Type 3 Diabetes Reflects Disordered Lipid Metabolism in the Human Brain Related to Higher Degree of Unsaturated Fatty Acids Composition and is not Related to Body Mass Index. J Bioanal Biomed 9: $159-163$. doi:10.4172/1948-593X.1000171

related to the aging process. Although some authors are pessimistic in the treatment of the cognitive impairment of T2DM elderly patients [28] our BMI data in combination with our lipidomics approach at postmortem neocortical tissue of the "Netherlands Brain Bank" [8] provides a new approach for pharmaceutical industry for the design of drugs and development of new nutritional therapies for neurodegenerative diseases in humans [29]. While it remains unclear whether management of T2DM [17] will reduce the incidence of Mild Cognitive Impairment (MCI) and mild-Alzheimer Disease (m-AD), emerging evidence suggests that diabetes therapies may improve cognitive function The use of pleiotropic drugs with multimodal mechanisms of action may have a role in the treatment of cognitive dysfunction and their use in order to prevent cognitive impairment would be warranted [12,13]. A role for pre-morbid ability in young adulthood as influencing the risk of both diabetes and cognitive impairment-one should think of quality and saturation degree of nutritional fats- has also been suggested [29].

\section{Acknowledgement}

Dr. M. Kooreman of the Netherlands Brain Bank, Netherlands Institute for Neuroscience, Amsterdam, Netherlands is kindly acknowledged for help with the selection of the patient material.

\section{References}

1. International Diabetes Atlas (2015) IDF diabetes atlas $\left(7^{\text {th }}\right.$ edn.)

2. Bordier L, Doucet J, Boudet J, Bauduceau B (2014) Update on cognitive decline and dementia in elderly patients with diabetes. Diabetes Metab 40: 331-337.

3. Sato N, Morishita R (2014) Brain alteriations and clinical symptoms of dementia in diabetes: $A \beta /$ Tau-dependent and independent mechanisms. Front Endocrinol (Lausanne) 5: 143

4. Sridhar GR, Lakshmi G, Nagamani G (2015) Emerging links between type 2 diabetes and Alzheimer disease. World J Diabetes 6: 744-751.

5. Sheen YJ, Sheu WH (2016) Association between hypoglycemia and dementia in patients with type 2 diabetes. Diabetes Res Clin Pract 116: 279-287.

6. Takeda S, Sato N, Rakugi H, Morishita R (2011) Molecular mechanisms linking diabetes mellitus and Alzheimer disease: beta-amyloid peptide, insulin signaling, and neuronal function. Mol Biosyst 7: 1822-1827.

7. Alzheimer's Association (2015) Alzheimer disease facts and figures. Alzheimers Dement 11: 332-384.

8. van Ginneken V, Verheij E, Hekman M, van der Greef J (2017) Characterization of the lipid profile post mortem for Type-2 diabetes in human brain and plasma of the elderly with LCMS-techniques: a descriptive approach of diabetic encephalopathy. Integr Mol Med 4: 1-10.

9. van Ginneken V, de Vries E, Verheij E, van der Greef J (2016) Metabolomics in hind limb- and heart muscle of a mouse model after a high-fat diet. Anat Physiol 6: 214

10. Rolfes SR, Pinna K, Whitney E (2009) Understanding normal and clinical nutrition. Wadsworth, Cengage Learning p: 925.

11. De la Monte SM, Wands JR (2008) Alzheimer disease is type 3 diabetesevidence reviewed. J Diabetes Sci Technol 2: 1101-1113.
12. Alagiakrishan K, Sankaralingam S, Ghosh M, Mereu L, Senior P (2013) Antidiabetic drugs and their potential role in treating mild cognitive impairment and Alzheimer's disease. Discove Med 16: 277-286.

13. Umegaki H (2016) Therapautic potential of antidiabetic medications in the treatment of cognitive dysfunction and dementia. Drugs Aging 33: 399-409.

14. Rodríguez-Gómez O, Palacio-Lacambra ME, Palasí A, Ruiz-Laza A, BoadaRovira M (2014). Prevention of Alzheimer's disease: a global challenge for next generation neuroscientists. J Alzheimer Dis 42: S515-S523.

15. Feinkohl I, Price JF, Strachan MW, Frier BM (2015) The impact of diabetes on cognitive decline: potential vascular, metabolic, and psychosocial risk factors. Alzheimers Res Ther 7: 46-68.

16. Anderson RJ, Freedland KE, Clouse RE, Lustman PJ (2001) The prevalence of comorbid depression in adults with diabetes: a meta-analysis. Diabetes Care 24: 1069-1078

17. Whitmer RA, Karter AJ, Yaffe K, Quesenberry CP, Selby JV (2009) Hypoglycemic episodes and risk of dementia in older patients with type 2 diabetes mellitus. JAMA 301: 1565-1572.

18. Abbatecola AM, Rizzo MR, Barbieri M, Grella R, Arciello A (2006) Postprandia plasma glucose excursions and cognitive functioning in aged type 2 diabetics. Neurology 67: 235-240

19. Korf ESC, White LR, Scheltens PH, Launer LJ (2006) Brain aging in very old men with Type-2 diabetes. The Honolulu-Asia Aging Study. Diabetes Care 29: 2268-2274.

20. Mijnhout GS, Scheltens P, Diamant M, Biessels GJ, Wessels AM (2006) Diabetic encephalopathy: a concept in need of a definition. Diabetologia 49: 1447-1448.

21. McGowan E, Eriksen J, Hutton M (2006) A decade of modeling Alzheimer's disease in transgenic mice. Trends Genet 22: 281-289.

22. Dimopoulos N, Piperi C, Salonicioti A, Psarra V, Mitsonis C (2007) Characterization of the lipid profile in dementia and depression in the elderly. $J$ Geriatr Psychiatry Neurol 20: 138-144.

23. van den Berg E (2006) Utrecht diabetic encephalopathy study group diabetic encephalopathy study group: Type 2 diabetes, cognitive function and dementia: vascular and metabolic determinants. Drugs Today 42: 741-754.

24. Yao JK, Leonard S, Reddy RD (2000) Membrane phospholipid abnormalities in postmortem brain from schizophrenic patients. Schizophr Res 42: 7-17.

25. Hulbert AJ, Turner N, Storlien LH, Else PL (2005) Dietary fats and membrane function: implications for metabolism and disease. Biol Rev 80: 155-169.

26. Salway JG (2006) Medical biochemistry at a glance. Wiley-Blackwell Publishing, UK.

27. Braak H, Braak E, Bohl J (1993) Staging of Alzheimer-related cortical destruction. Eur Neurol 33: 403-408.

28. Bornstein NM, Brainin M, Guekht A, Skoog I, Korczyn AD (2014) Diabetes and the brain issues and unmet needs. Neurol Sci 35: 995-1001.

29. Keller JN (2009) Reciprocal interactions between diet, metabolism and the nervous system. Biochim Biophys Acta 1792: 393-394. 